\title{
Mantenimiento y cambio lingüístico: fricativas sibilantes en gallego*
}

\author{
Xosé Luís RegUEIRA \\ Instituto da Lingua Galega \\ Universidade de Santiago de Compostela \\ xoseluis.regueira@usc.es
}

\begin{abstract}
RESUMEN
La lengua de las comunidades rurales se encuentra sometida a grandes presiones de cambio, tanto por la presencia de la lengua estándar como, en el caso de Galicia, de la lengua española. Entre los cambios lingüísticos destacan algunos que se refieren a la fonética y a la fonología y que van en la dirección de converger con el estándar gallego y, en parte, con el español. En este trabajo vamos a explorar una de estas variedades rurales, que presenta un sistema de sibilantes diferente del estándar gallego y del español, a través del análisis de las sibilantes de dos grupos de hablantes de localidades muy próximas entre sí, con personas de dos grupos de edad en cada una de ellas, trataremos de determinar si se detectan algunos de los cambios que se describen en estudios anteriores, en qué medida y en qué dirección, así como si se perciben resistencias al cambio en los miembros de los grupos más jóvenes.
\end{abstract}

Palabras clave: lengua gallega, cambio fonético, lengua estándar, sibilantes.

[Recibido, septiembre 2013; aprobado, diciembre 2013]

Language maintenance and change:

fricative sibilants in Galician

\begin{abstract}
The language of rural communities is under great pressure to change, both by the presence of the standard language as, in the case of Galicia, of the Spanish language. Among the linguistic changes involved, some concern the phonetics and the phonology and go in the direction of convergence with standard Galician and, in part, with Spanish. In this paper we will explore one of these rural varieties, which presents a set of sibilant different from Galician Standard and Spanish, through the analysis of the sibilants of two groups of speakers from nearby places, with people from two age groups in each place, we will try to determine whether some of the changes described in previous studies take place, to what extent and in what direction are detected, and whether it exists a perceived resistance to change in the members of the youngest groups.
\end{abstract}

Keywords: Galician language, phonetic change, standard language, sibilants.

\footnotetext{
* Este trabajo ha sido realizado en el marco del proyecto «Cambio lingüístico en el gallego actual», financiado por el Ministerio de Economía y Competitividad (Ref FFI2012-33845).
} 


\section{Introducción}

La lengua de las comunidades rurales se encuentra sometida a grandes presiones de cambio, tanto por la presencia de la lengua estándar como, en el caso de Galicia, de la lengua española. Entre los cambios lingüísticos que se han descrito, destacan algunos que se refieren a la fonética y a la fonología (Regueira 2009), y que van en la dirección de converger con el estándar gallego y, en parte, con el español.

En este trabajo vamos a explorar una de estas variedades rurales, que presenta un sistema de sibilantes diferente del estándar gallego y del español en los fonemas (/s/ y / $/$ frente a $/ \theta /, / \mathrm{s} / \mathrm{y} / \mathrm{s} /$; es decir, se trata de una variedad carente de $/ \theta /$, o de «seseo»), y también es diferente en las realizaciones, ya que presenta fricativas laminoalveolares [s], a veces laminodentales [s] , así como apicopostalveolares [s], que son sonidos ausentes tanto del estándar gallego como del español de Galicia.

A través del análisis de las sibilantes de dos grupos de hablantes de localidades muy próximas entre sí, con personas de dos grupos de edad en cada una de ellas, trataremos de determinar si se detectan algunos de los cambios que se describen en estudios anteriores (González 1991), en qué medida y en qué dirección, así como si se perciben resistencias al cambio en los miembros de los grupos más jóvenes.

Los resultados obtenidos serán de interés tanto para el estudio del cambio lingüístico del gallego como para la comprensión de los factores que intervienen en estos procesos en comunidades rurales, en una sociedad que ya está altamente urbanizada y en la que las estructuras productivas tradicionales han desaparecido para dar paso a una economía basada en la producción para la industria y la comercialización.

El presente trabajo forma parte de un proyecto de investigación más amplio y consta todavía de un número de datos insuficiente para extraer conclusiones definitivas, pero creemos que el análisis cualitativo de un número relativamente importante de casos puede aportar indicios que, junto con otros, serán suficientes para establecer líneas de cambio o de resistencia y mantenimiento de una variedad lingüística diferenciada e identificadora.

\section{Cambio lingüístico en gallego: factores internos, contacto lingüístico y cam- bio sociocultural}

La situación lingüística en Galicia reúne condiciones que la hacen especialmente interesante para el estudio de la variación y el cambio lingüístico. Por una parte se produce un contacto generalizado entre gallego y castellano que afecta, en mayor o menor medida, a toda la sociedad. Por otra parte, la lengua estándar se ha extendido a través del sistema escolar y de los medios de comunicación, principalmente de la radio y la televisión. Las hablas autóctonas se encuentran sometidas, por tanto, a la doble superposición de variedades lingüísticas más prestigiosas, lo que hace que se produzcan cambios orientados hacia el gallego estándar (visibles sobre todo en el léxico, pero también en la gramática y la fonética), y otros afectados por el español, que sigue siendo la lengua de mayor prestigio (González et al. 2003; Iglesias 2002).

Históricamente el gallego se mantuvo como lengua de la inmensa mayoría de la población, a pesar de que el español fue la lengua única del poder desde el siglo XV hasta 1981, año en que se aprobó el Estatuto de Autonomía de Galicia, que declara oficial la lengua gallega, junto con la castellana. Todavía a principios del S. XX se estima que hablaba gallego de manera habitual más del $90 \%$ de la población, pero ese porcentaje se fue reduciendo progresivamente, y en 1992 era del 68,6\% (MSG 1992). En la 
actualidad la pérdida de transmisión de padres a hijos sigue produciéndose: entre 1992 y 2004 el uso del gallego como lengua habitual en la población de entre 15 y 54 años cayó del 53\% hasta el 38,4\% (MSG 2004).

La resistencia de las lenguas autóctonas ante las lenguas del poder es un fenómeno constatado en diferentes lugares (cfr. Nettle \& Romaine 2000: 133-143), y obedece a lo que Labov llamó "prestigio encubierto". En estas sociedades, fundamentalmente rurales y marineras, con redes sociales bien trabadas, que mantenían la lengua y la cultura propias, la movilidad social era reducida, y los beneficios de cambiarse a la lengua del poder eran escasos.

La sociedad gallega, en la que se mantenía una diglosia social (Rojo 1981) con el español como lengua del poder, pero con el gallego bien instalado y protegido por la densidad de las redes sociales, se transformó profundamente y de manera acelerada, en un proceso del tipo de los que Dixon (1997) llamó punctuation (interrupción): las redes sociales se rompieron debido a la emigración y al éxodo a las ciudades, los métodos de trabajo tradicionales se abandonaron y la producción agraria y pesquera se industrializó, la cultura de transmisión oral se interrumpió, las costumbres y mentalidades se urbanizaron y penetraron los medios de comunicación de masas (Roux 1982: 145; Regueira 2012: 30).

Las comunidades rurales, por tanto, en contra de la concepción que muchas veces se ofrece de ellas, no se encuentran aisladas, sino sometidas a intensos procesos de cambio. Como señala Medeiros (2006: 305-313),las comunidades aparentemente más aisladas han estado y están en contacto directo y frecuente con los centros urbanos e industriales de Europa y de América a través de la emigración. En los últimos tiempos, además, el modo de vida se ha urbanizado, son frecuentes las visitas a la ciudad para realizar compras y gestiones de todo tipo, la gente joven va a estudiar a un centro urbano, por lo que el contacto cultural y lingüístico se ha acrecentado de un modo extraordinario.

Todo esto tiene, sin duda, efectos notables sobre la lengua y la comunicación oral. La sociedad ha cambiado radicalmente, las relaciones familiares, afectivas y sociales ya no corresponden a las que existían en la cultura tradicional de hace unas décadas. Son bien visibles los cambios en el léxico y la fraseología, pero creemos que también afectan a la gramática y a la pronunciación. Estos factores externos vienen a sumarse a algunos procesos de cambio que ya habían sido observados y que parecen obedecer a razones internas, sobre todo en el terreno de la fonología y la fonética (Regueira 2009).

En este trabajo estudiamos la realización de las fricativas sibilantes en dos grupos de hablantes del occidente de la provincia de A Coruña: 3 hombres de Corzón (ayuntamiento de Mazaricos) y 6 mujeres de Dumbría (ayuntamiento de Dumbría). Los dos lugares, situados a 12,5 Km de distancia, pertenecen a una misma área lingüística (área fisterrá, cfr. Fernández Rei 1991: 110), caracterizada por un sistema de fricativas informalmente conocido como de seseo predorsal, es decir, que carece de $/ \theta$ / y que presenta una sibilante descrita como fricativa predorsodentalen los estudios de dialectología derivados del Atlas Lingüístico Galego (ALGa), como Fernández Rei (1991) o González (1991), pero que sería más exacto describir como laminoalveolar (Vidal 1993). En esta área se han detectado algunos cambios que afectan a estos sonidos, y que describiremos en el apartado siguiente.

Los dos grupos de informantes están repartidos en dos grupos de edad: uno de personas mayores ( 3 mujeres de 45, 73 y 73 años; 1 hombre de 64 años) y otro de gente más joven ( 3 mujeres de 22, 24 y 26 años; 2 hombres de 30 y 35 años). Dos de las 
hablantes jóvenes son hijas de la informante de 45 años, y la otra es hija de una de las mujeres de 73. Esa fue la razón de que incluyéramos a la informante de 45 años, a pesar de la notable diferencia de edad con las otras dos, ya que nos pareció de interés disponer de datos de madres e hijas. El grupo masculino muestra, en comparación, un mayor desequilibrio, al incluir solo un hablante mayor y dos jóvenes (que también son parientes entre sí). No obstante, nuestro interés en este trabajo es fundamentalmente cualitativo, no cuantitativo, por lo que ese factor no invalida los resultados que obtengamos.

Los dos grupos seleccionados presentan además diferencias socioculturales notables en las generaciones jóvenes, ya que los hombres viven y trabajan en el lugar, y su actividad se centra en la producción lechera, como copropietarios de una moderna explotación con un importante número de cabezas de ganado, mientras que las mujeres jóvenes tienen estudios universitarios, y estudian y trabajan durante la semana en Santiago de Compostela. No obstante, muestran una actitud consciente de mantenimiento de su variedad lingüística, al menos en sus rasgos más característicos (gheada, seseo), y se sienten muy ligadas a su comunidad de origen. Por el contrario, las personas mayores se han dedicado o se dedican todas ellas fundamentalmente a la ganadería, aunque el hombre de 64 años también pasó algún tiempo como obrero de la construcción en Euskadi y en Liechtenstein.

\section{Fricativas sibilantes en gallego: estabilidad y cambio}

El sistema de fricativas(sin tener en cuenta la gheada)de la mayoría de las hablas gallegas consta de tres unidades distintivas: / $\theta /$ (caza) - /s/ (casa) - / $/$ / (caixa); en final de palabra aparecen dos: / $\theta /(v o z)$ - /s/ (vós), aunque en casi la mitad occidental del territorio se produce una neutralización y solo se registra la fricativa /s/ (Fernández Rei 1991: 193). Este sistema con $/ \theta /$ es el de la lengua estándar que se utiliza en la educación, en los medios de comunicación y en los demás ámbitos de la actividad pública. También coincide en lo fundamental (la diferencia contrastiva entre $/ \theta / \mathrm{y} / \mathrm{s} /$, así como en sus realizaciones fonéticas) con el español de Galicia y del norte de España en general (Martínez Celdrán \& Fernández Planas 2007: 110).

El área que nos ocupa, en cambio, corresponde a lo que Fernández Rei (1991: 193) denomina «galego con seseo predorsodental», con solo dos sibilantes: /s/ (caza, casa) $/ \mathrm{J} /$ (caixa); en final de palabra aparece generalmente la realización apicoalveolar [s] (voz, vós), aunque en algunos puntos aparece $[\mathrm{s}]$. Existe también una variante: $/ \mathrm{s} /-/ \mathrm{S} / \sim$ $/ \mathrm{s} /$,con [s] en fin de palabra.

Este sistema muestra diferentes procesos de cambio que, de forma interesante, no parecen ir en la dirección de convergencia con el estándar (y el español). En algunos casos puede simplificarse, de modo que las dos sibilantes contrastivas se reducen a una sola. La primera descripción de estos procesos fue realizada por González (1991), a partir de los datos del ALGa, recogidos entre los años 1974 y 1976 (el volumen de fonética fue publicado en 1999). En ese trabajo, González indica que, en algunos hablantes, la fricativa laminopostalveolar [ $\left.\int\right]$ ha pasado a realizarse como apicopostalveolar, representada como [s] (Vidal 1993), o bien como apicoalveolar [s], lo cual aparece recogido en 18 puntos del ALGa. De este modo, se registró un sistema de dos fricativas sibilantes /s/ - /s/ (caza, casa / caixa), junto a otro que consta de un único fonema /s/. Ambos sistemas, según González (1991: 547), «están identificados con grupos sociais diferenciados pola idade». Estos cambios fueron constatados en algunas otras grabaciones realizadas en la zona. 
En el punto del ALGa más próximo a los lugares encuestados por nosotros (C33, A Picota, a 4,5 km de Corzón y a 16 de Dumbría), González (1991: 545) registra el sistema de dos sibilantes /s/ - /s/ «na fala das xeracións novas e de idade media». A pesar de que han transcurrido casi 40 años entre los datos del ALGa y nuestras grabaciones, no hemos encontrado este sistema, ni tampoco la simplificación a una sola fricativa, aunque sí hemos podido constatar la inestabilidad de las realizaciones sibilantes, y la existencia de cambios que configuran una situación más compleja.

\section{Metodología}

El presente trabajo se basa en la grabación de una encuesta sobre fricativas sibilantes realizada por dos grupos de hablantes de Corzón (Mazaricos), y de Cheo (Dumbría), como hemos dicho más arriba (apartado 2), en la que las respuestas fueron elicitadas mediante dibujos. El objetivo era obtener muestras de la realización de las diferentes sibilantes en posición inicial y medial entre las tres vocales extremas /i, a, u/ y las vocales medias anteriores y posteriores /e, $\varepsilon /, / \mathrm{o}, \mathrm{o} /$ respectivamente. También se buscaron las realizaciones de las fricativas en posición final de palabra. Cada palabra fue repetida dos veces. En algunos casos no hemos podido obtener ciertos ejemplos, por encontrar respuestas no previstas (como en zanco, que los hablantes de Corzón denominan coxón, coxote), o bien por la presencia de préstamos del castellano (como girar, en lugar de xirar). Como resultado se obtuvieron 426 ejemplos en el grupo femenino y 182 en el masculino.

Las respuestas fueron registradas en una grabadora Marantz PDM 671, con un micrófono Shure SM10A. Las grabaciones se realizaron con una frecuencia de muestreo de $24 \mathrm{KHz}, 16$ bits. El análisis acústico fue efectuado con la plataforma Multi-Speech 3700 de Kay Elemetrics. De cada ejemplo se obtuvo un espectro de 1024 puntos de la zona central de la fricativa, para reducir el efecto de la coarticulación, de lo que resultan 512 valores de energía entre 0 y $12 \mathrm{KHz}$. Para cada espectro se calculó la media espectral (centro de gravedad o centroide) y la asimetría. También se realizaron LPC con 6 polos, en una ventana Blackman de $30 \mathrm{~ms}$ tomada del centro de la fricativa, con lo que se obtuvieron 3 o 2 picos, de los que tuvimos en cuenta la frecuencia y el ancho de banda. Los datos fueron analizados para comprobar la significatividad de las diferencias entre fricativas, entre informantes y entre grupos de edad, con el software estadístico $\mathrm{R}$ (R Core Team 2013).

Ciertamente el estudio de las fricativas ha sido y es una de las cuestiones más complejas de la fonética acústica. Se han intentado diferentes aproximaciones, con resultados a menudo poco satisfactorios, sobre todo para fricativas como [f] y [ $\theta]$. En cuanto a las sibilantes, varios estudios han mostrado que la distinción entre [s] y [S] se encuentra en el ruido de fricción (Hughes \& Halle 1956; Evers et al. 1998, entre otros).

El estudio más completo y detallado que se ha realizado de las fricativas del gallego hasta el momento es el de Labraña (2005), que utiliza espectrogramas y LPC de 6 polos. En nuestro estudio los datos extraídos de los LPC permitieron distinguir con cierta seguridad las fricativas $/ \mathrm{J} /$, /s/ y también la fricativa en posición final, más grave. No obstante, ni los resultados de frecuencia para los picos del LPC ni su ancho de banda arrojaron resultados que permitiesen contrastar de forma significativa las diferentes realizaciones $\mathrm{de} / \mathrm{s} /$ en los distintos hablantes o grupos.

Por tanto, nos hemos centrado en el estudio de los espectros, a través de la comparación directa, y sobre todo del análisis de las medias espectrales y de la asimetría, que 
se han revelado como índices significativos para distinguir fricativas en diferentes estudios (Nittrouer et al. 1989; Jongman et al. 2000; Gordon et al. 2002). La idea de partida es que la cavidad posterior a la constricción no tiene un papel relevante en las sibilantes, pero sí las cavidades anteriores; como afirman Evers etal. (1998), «the resonances of the vocal tract in front of the constriction determine the frequencies of the spectral peaks. With a more anterior constriction, as for [s], the front cavity is smaller and hence the resonance frequencies are higher. In contrast, for [f], which has a more posterior constriction, the resonance frequencies are somewhat lower». Por tanto, si cuanto más anterior es la constricción más agudas son las resonancias, podremos comprobar si estos parámetros nos permiten también diferenciar diferentes realizaciones alveolares y dentales.

Aunque en los LPC se muestran diferencias entre contexto de vocales labializadas y no labializadas (también en Labraña 2005), no lo hemos tenido en cuenta por razones de espacio y también porque en todos los hablantes la relación entre contexto labializado y no labializado es la misma (40\% labializado, $60 \%$ no labializado), por lo que, al realizar el promedio de todas las realizaciones de una fricativa, las comparaciones entre diferentes hablantes no se ven afectadas por este hecho.

Las comparaciones se establecerán preferentemente entre hablantes de cada grupo de sexo, que también comparte lugar de nacimiento y residencia. Esto se debe a que en diferentes estudios se ha constatado que existen diferencias significativas entre hombres y mujeres en la realización de sonidos fricativos, especialmente de sibilantes. En un estudio pionero, Schwartz (1968) demostró en un estudio perceptivo que los oyentes podían distinguir el sexo de la persona que había producido una sibilante a partir de la audición de ejemplos aislados de fricativas sordas. Los ejemplos emitidos por mujeres presentaban frecuencias más elevadas que los realizados por hombres. En estudios posteriores se encontraron diferencias espectrales entre las sibilantes producidas por hablantes de sexo femenino y masculino (p.e. Jongman et al 2000: 1256; Gordon et al. 2002: 144). Habitualmente se atribuyen estas diferencias al tamaño de la cavidad bucal, es decir, a factores biológicos, pero se ha mostrado que las diferencias obedecen también a factores sociofonéticos, es decir, sociales y culturales (Fuchs \& Toda 2010; Munson et al. 2006).

\section{Resultados}

Analizamos en primer lugar las diferencias entre los grupos de sexo, y posteriormente entre los grupos de edad dentro de cada grupo de sexo.

\subsection{Diferencias entre grupos de sexo}

En nuestra muestra las realizaciones femeninas presentan resultados más agudos en los picos obtenidos por el LPC. En el conjunto de datos para todas las realizaciones fricativas, las medias para los tres picos femeninos están situadas a $4126.55,6732.70 \mathrm{y}$ 9024.46, mientras que las medias masculinas se quedan en frecuencias más bajas, especialmente en el primer pico: $3319.90,6467.53$ y 8657.56 .

También hemos obtenido diferencias significativas en el centro de gravedad de los espectros femeninos y masculinos. El conjunto de fricativas femeninas obtiene una media de $5475 \mathrm{~Hz}$ (95\% intervalo de confianza $5320-5630 \mathrm{~Hz})$, mientras que la masculina es de $4598 \mathrm{~Hz}(95 \%$ intervalo de confianza 4361-4836 Hz). La prueba t de Student arroja un valor $\mathrm{t}=6.07, \mathrm{p}<.0001$. Las diferencias entre hombres y mujeres se muestran 
significativas en todos los sonidos analizados, así como en las comparaciones por grupos de edad (hombres-mujeres de generaciones jóvenes y mayores). En la Tabla 1 vemos los resultados totales y los correspondientes a las fricativas [s] y [S] en posición inicial de sílaba $(\mathrm{M}=$ mujeres, $\mathrm{H}=$ hombres; 1 = grupo joven, 2 = grupo mayor):

\begin{tabular}{|l|l|l|l|l|l|l|l|}
\hline Mujeres & $\begin{array}{l}\text { Media } \\
\text { Hz }\end{array}$ & $\begin{array}{l}\text { Intervalo } \\
\text { confianza } \\
95 \%\end{array}$ & Hombres & $\begin{array}{l}\text { Media } \\
\mathrm{Hz}\end{array}$ & $\begin{array}{l}\text { Intervalo } \\
\text { confianza } \\
95 \%\end{array}$ & $\begin{array}{l}\text { t de } \\
\text { Student }\end{array}$ & probabilidad \\
\hline$[\mathrm{s}] \mathrm{M} 2$ & 6569 & $6170-6968$ & {$[\mathrm{~s}] \mathrm{H} 2$} & 4948 & $4231-5665$ & $\mathrm{t}=3.92$ & $\mathrm{p}=0.0002$ \\
\hline$[\mathrm{s}] \mathrm{M} 1$ & 6579 & $6372-6786$ & {$[\mathrm{~s}] \mathrm{H} 1$} & 6172 & $5919-6425$ & $\mathrm{t}=2.46$ & $\mathrm{p}=0.015$ \\
\hline$[S] \mathrm{M} 2$ & 3971 & $3681-4260$ & {$[S] \mathrm{H} 2$} & 3160 & $2658-3661$ & $\mathrm{t}=2.83$ & $\mathrm{p}=0.0073$ \\
\hline$[S] \mathrm{M} 1$ & 4737 & $4500-4973$ & {$[S] \mathrm{H} 1$} & 3515 & $3189-3840$ & $\mathrm{t}=6.05$ & $\mathrm{p}=<.0001$ \\
\hline
\end{tabular}

Tabla 1. Resultados de la comparación entre las realizaciones masculinas y femeninas totales y separadas por grupos de edad.

Dados estos resultados, las comparaciones de nuestro corpus se establecerán dentro de cada grupo de sexo, lo que por otra parte asegura que no intervienen factores de diferencia dialectal, ya que cada grupo pertenece a un lugar diferente, aunque están muy próximos y ellos mismos aseguran que no existen diferencias entre el habla de Dumbría y la de Mazaricos.

\subsection{Contraste entre fricativas}

En primer lugar hemos comparado los espectros (la media de todas las realizaciones) de las diferentes fricativas dentro de cada grupo de sexo y de edad. En la Fig. 1 se muestran los resultados obtenidos para el grupo femenino joven (M1):

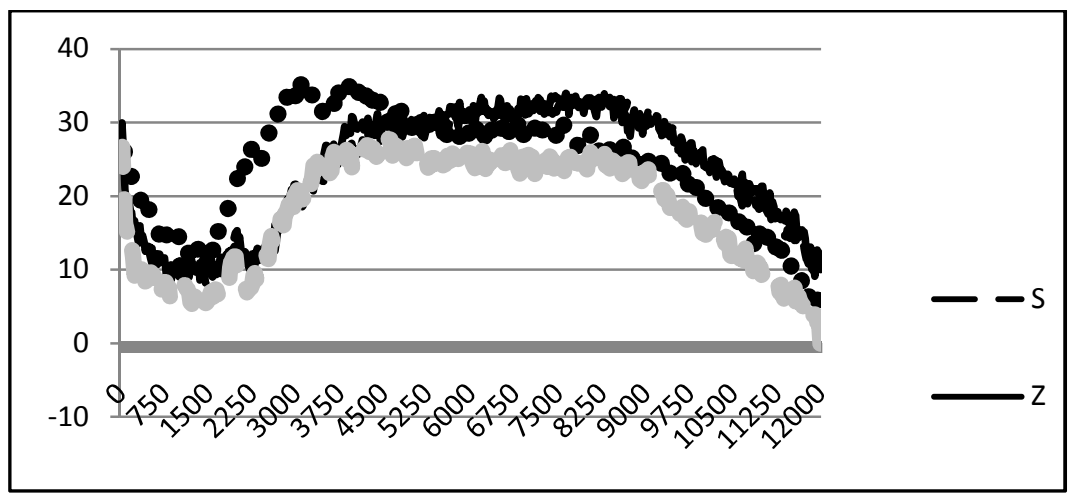

Figura 1. Espectros medios del grupo M1.

Como puede verse en este gráfico, las fricativas que corresponden a los fonemas del gallego estándar $/ \mathrm{s} /$ y $/ \theta /$ presentan una realización fonética idéntica (aquí representadas por las letras con que se escriben, $s$ y $z$, por razones de claridad); se observa que las líneas de los espectros (promedio de 56 y de 54 espectros, respectivamente) se superponen de manera casi perfecta. En cambio la fricativa [ $\left.\int\right]$ (representada por $x$, prome- 
dio de 53 espectros) se diferencia claramente por presentar altas intensidades a frecuencias más bajas, entre 2 y $4 \mathrm{KHz}$, mientras que las realizaciones de [s] se elevan a partir de $3,5 \mathrm{KHz}$ y continúan subiendo, en una curva sostenida, hasta llegar casi a los $9 \mathrm{KHz}$.

Como es de esperar, los centros de gravedad de las fricativas con la etiqueta $s$ y $z$ son casi idénticos (6583 y $6.575 \mathrm{~Hz}$, respectivamente), con un valor en la prueba de Student det $=0.378$, con $\mathrm{p}=0.97$. En cambio el contraste entre los espectros de $[\mathrm{s}]$ y los de $x$ son claros: la media espectral de las realizaciones de [s] es de $6583 \mathrm{~Hz}$, frente a 4737 Hzpara [ $]$ ], $\mathrm{t}=9.80$ y $\mathrm{p}<.0001$. También la asimetría presenta valores significativos para estas dos realizaciones, con valores de -0.356 para [s] y 0.728 para [S], lo que arroja un valor en la prueba de Student de $\mathrm{t}=-5.34$, con $\mathrm{p}<.0001$.

Esta situación se repite en todos los grupos, con diferencias consistentes entre la fricativa $\left[\int\right]$ y $[\mathrm{s}]$, pero no entre las realizaciones de $[\mathrm{s}]$ entre los casos en que se corresponden a $[\theta]$ ou a $[\mathrm{s}]$ en las hablas no seseantes. Como muestra, podemos ver lo que sucede en el grupo joven masculino, que presenta una curva diferente para la fricativa anterior, con una pronunciación laminoalveolodental o laminodental, y que tiene su máxima elevación en frecuencias muy altas (alrededor de los $8,5 \mathrm{KHz}$ ), mientras que la fricativa [S] empieza a presentar energía alta a partir $1,5 \mathrm{KHz}$ y cae a partir de $5 \mathrm{KHz}$.La diferencia entre los centros de gravedads y $z$ se muestra no significativa $(\mathrm{p}=0.14)$, mientras que los centroides de[s] y [S] (con valores medios de 6344 y $3515 \mathrm{~Hz}$, respectivamente) presentan un valor $\mathrm{t}=13.3, \mathrm{p}<.0001$.

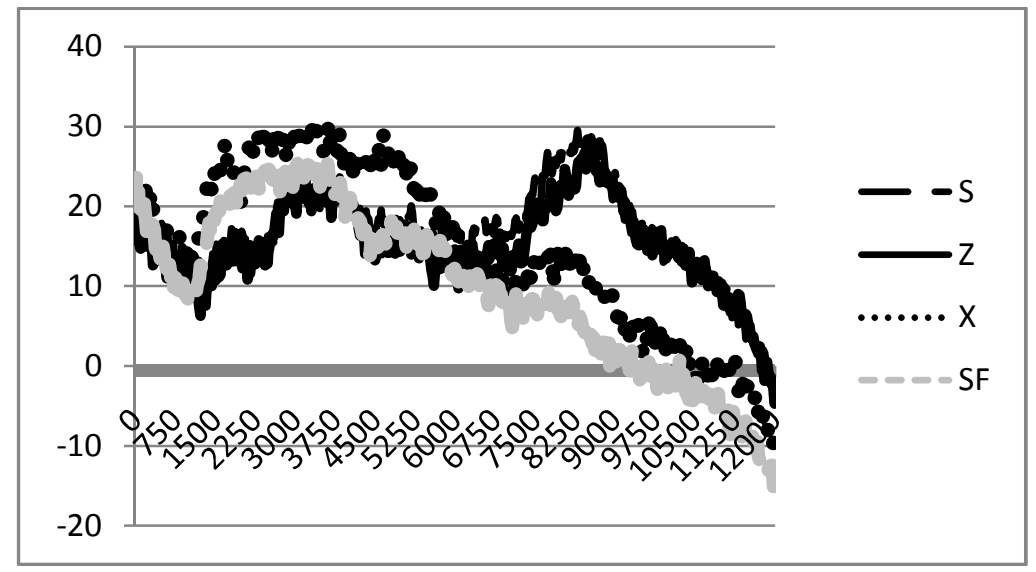

Figura 2. Espectros medios del grupo H1.

En lo que se refiere a las realizaciones de la fricativa en posición final, representadas por una línea gris claro en los gráficos 1 y 2 , también presentan valores significativamente diferentes. En el grupo femenino (Fig. 1), sigue un perfil similar al de [s], aunque a partir de $3,5 \mathrm{KHz}$ presenta una energía significativamente menor. La comparación de las series de valores de los espectros entre estas dos fricativas arrojan un valor $t$ de Student de 5.23, con $\mathrm{p}<.0001$, ya que las realizaciones finales tienen una media espectral más grave $(5538 \mathrm{~Hz}$ de promedio, frente a $6583 \mathrm{~Hz}$ para [s]). También es significativa la asimetría (media de 0.394 frente a -0.356 para $[\mathrm{s}], \mathrm{t}=3.27, \mathrm{p}=0.0014$ ). En el grupo masculino, en cambio, esta fricativa sigue un perfil semejante al de [f], aunque con menor energía en casi todo el recorrido, y los centros de gravedad de estas realiza- 
ciones son significativamente más bajos que los de [J] $(2765 \mathrm{~Hz}$, frente a $3515 \mathrm{~Hz}$ para $\left.\left[\int\right], \mathrm{t}=-4.47, \mathrm{p}<.0001\right)$. En este caso, dado que las dos curvas tienen un perfil muy similar, la asimetría no resulta un índice significativo (media 0.639 para [J], 1.51 para la fricativa en posición final, $\mathrm{t}=1.97, \mathrm{p}=0.054$ ).

Estas pruebas muestran que los centros de gravedad y la asimetría pueden diferenciar de manera consistente las realizaciones de [s] y de [J], del mismo modo que ocurría en otros estudios previos (Nittrouer et al. 1989; Gordon et al. 2002) en diferentes lenguas no relacionadas, pero también otras realizaciones, como las que aparecen en estas hablas en posición final de sílaba.

\subsection{Grupos de edad}

En la comparación entre los grupos de edad, tanto masculinos como femeninos, las diferencias más notables e interesantes se producen en las realizaciones de las fricativas alveolares [s] o laminodentales [s] , así como en las realizaciones en posición final de palabra. Esto coincide con otros estudiosen los que se ha comprobado que estos sonidos presentan una gran variabilidad entre lenguas y también entre hablantes de una misma lengua; como dicen Gordon et al. (2002: 170), esta variación sugiere «interspeaker and interlanguage differences in location of constriction, constriction length, and tongue body height».

Por el contrario, las realizaciones de la fricativa postalveolar [ $]$ ] presentan una notable consistencia. En el grupo masculino no se aprecia diferencia en la pronunciación de [S] entre los dos jóvenes y el hablante mayor, con curvas muy similares y valores relativamente próximos de las medias espectrales y en la asimetría: en los jóvenes (H1) 3515 $\mathrm{Hz}$, frente a 3160 en $\mathrm{H} 2(\mathrm{p}=0.14)$ para los centros de gravedad, y 0.652 frente a 0.590 en asimetría $(\mathrm{p}=0.84)$ :

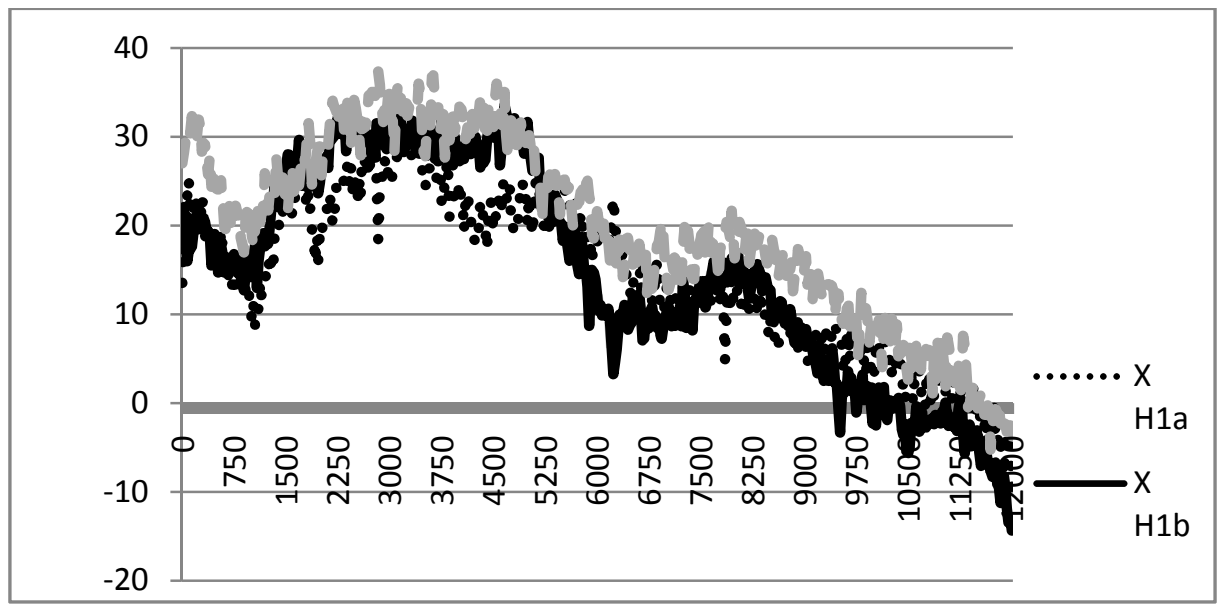

Figura 3. Curvas espectrales para $\left[\int\right]$ en hablantes masculinos.

En el grupo femenino, la situación se asemeja, aunque con algunas diferencias. La curva del grupo joven (M1) se eleva un poco sobre la del grupo mayor (M2), pero se trata de un efecto producido por una de las hablantes jóvenes (M1M), que presenta energías más altas en las frecuencias medias y elevadas respecto de las otras hablantes. 
Si no consideramos las realizaciones de esa hablante, las curvas coinciden casi perfectamente, sobre todo a partir de los 2,5 KHz. Las curvas de tendencia polinómicas (orden 6) se superponen. No obstante, la mayor energía en la baja frecuencia en el grupo de edad 2 provoca una diferencia en los centros de gravedad (M2 $3854 \mathrm{~Hz} / \mathrm{M} 14432 \mathrm{~Hz}$ ), que estadísticamente resulta significativa $(\mathrm{t}=3.19, \mathrm{p}=0.0020)$.

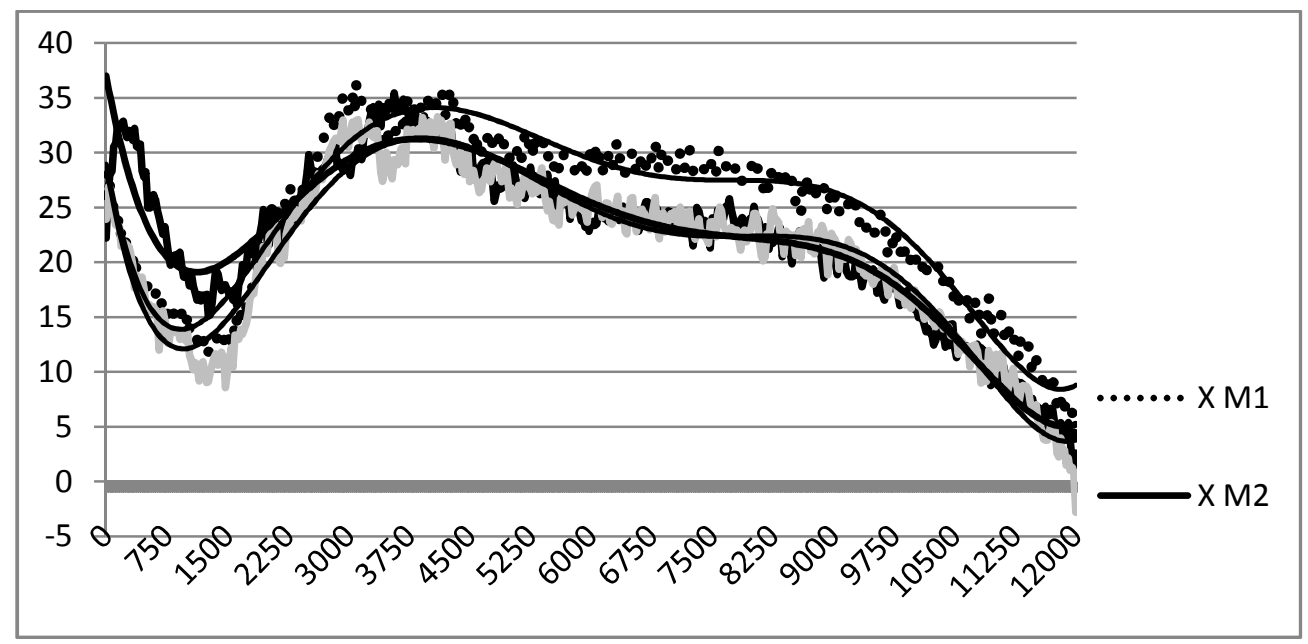

Figura 4. Curvas espectrales para [S] y líneas de tendencia polinómica (orden 6) en hablantes femeninas.

En cuanto a las realizaciones de /s/ en posición inicial de sílaba, la situación es bastante diversa en los dos lugares. Por una parte, en Corzón nos encontramos con dos realizaciones diferentes, según el grupo de edad:

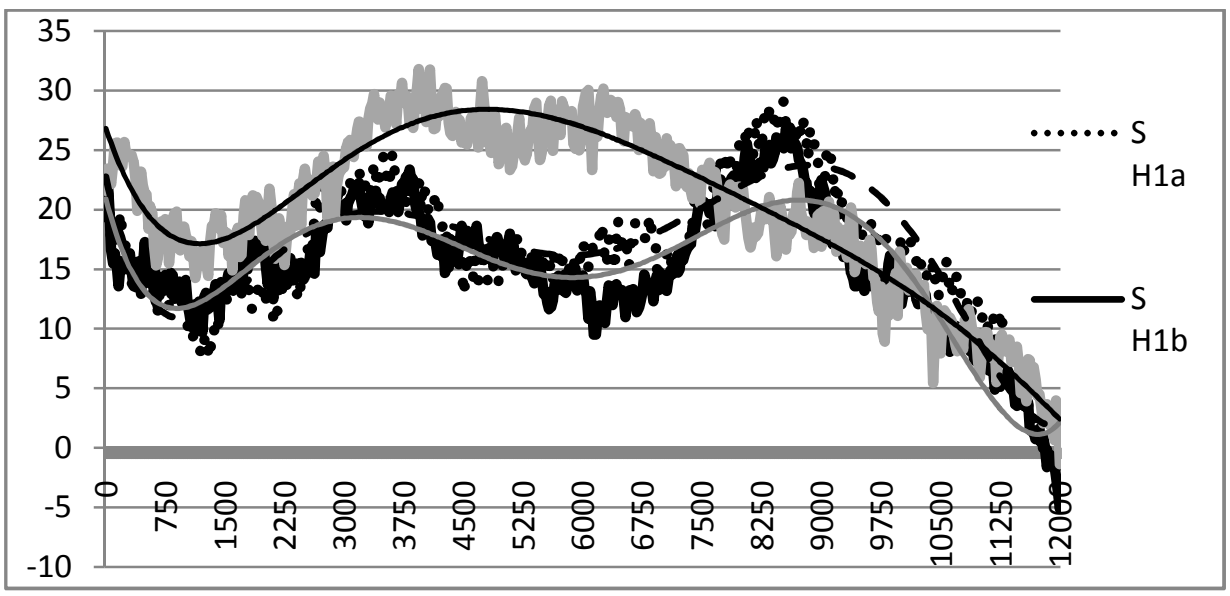

Figura 5. Curvas espectrales para las realizaciones de /s/ y líneas de tendencia polinómica (orden 6) en hablantes masculinos. 
En la Figura 5 se ve que los hablantes jóvenes (H1a y H1b) presentan una fricativa cuyo máximo de energía se halla situado en frecuencias muy altas, entre los 8 y los 8,5 $\mathrm{KHz}$, tras un pico inicial alrededor de los $3,5 \mathrm{KHz}$. Obsérvese la coincidencia de las líneas de tendencia polinómica (orden 6), que contrastan con la correspondiente al hablante de más edad (H2). En esta curva espectral, los máximos están situados entre los 3,5 y los 5,5 KHz. En cuanto a los centros de gravedad, las realizaciones de los hablantes del grupo joven (H1) no presentan diferencias estadísticamente significativas (medias de 6614 y $6061 \mathrm{~Hz}, \mathrm{p}=0.071$ ), ni tampoco lo hacen los valores de asimetría (0.675 y $-0.408, \mathrm{p}=0.19$ ). Pero sí los de cualquiera de ellos, o de la media de ambos, con respecto a H2: 6344 frente a $4948 \mathrm{~Hz}, \mathrm{p}<.0001$. También la asimetría es significativamente diferente: $-0.549(\mathrm{H} 1) /-0.114(\mathrm{H} 2), \mathrm{p}=0.032$.

En el grupo femenino, la comparación entre las tres hablantes mayores revela perfiles diferentes en los espectros, como se ve en la Figura 6:

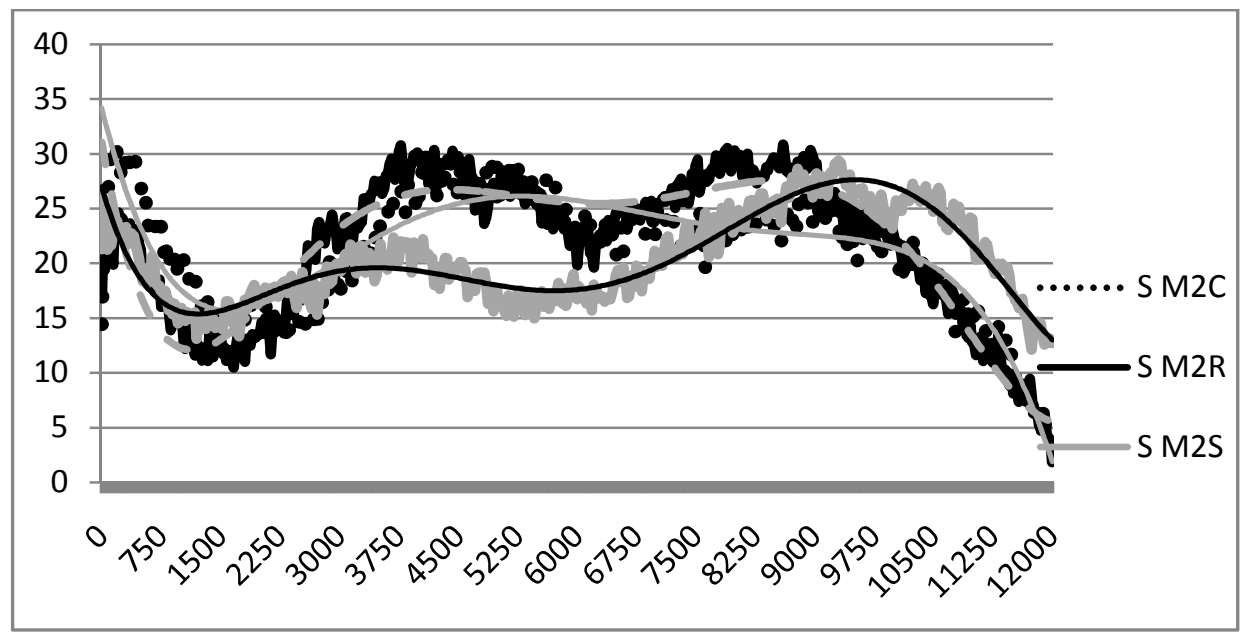

Figura 6. Curvas espectrales para las realizaciones de /s/ y líneas de tendencia polinómica (orden 6) en el grupo M2.

En la Fig. 6 se puede observar que la hablante M2S (línea gris) se separa de las otras dos, y presenta un espectro medio similar al de los jóvenes de Corzón, con máximos entre 8.5 y $10.5 \mathrm{KHz}$. Este tipo de realización corresponde a una realización muy adelantada, laminoalveolodental o laminodental, que contrasta con las de sus vecinas, que presentan igualmente alta energía en frecuencias muy agudas (entre $7.5 \mathrm{y} 9 \mathrm{KHz}$ ), pero también en frecuencias más graves $(3.5-5 \mathrm{KHz})$. No obstante también entre estas existen algunas diferencias, dado que la cantidad de energía en altas frecuencias es más elevada en la hablante M2R (centroide medio $5948 \mathrm{~Hz}$ ) que en M2C (4438 Hz), y la series son estadísticamente diferentes $(\mathrm{p}=0.0003)$. Frente a ellas, la media para M2S es de $7172 \mathrm{~Hz}$, mucho más elevada.

De hecho, si superponemos las curvas de esta hablante con las de los jóvenes de Corzón, podemos observar que las líneas de tendencia de las tres medias espectrales siguen un curso muy similar, aunque la realización femenina se mantiene alta hasta frecuencias más agudas: 


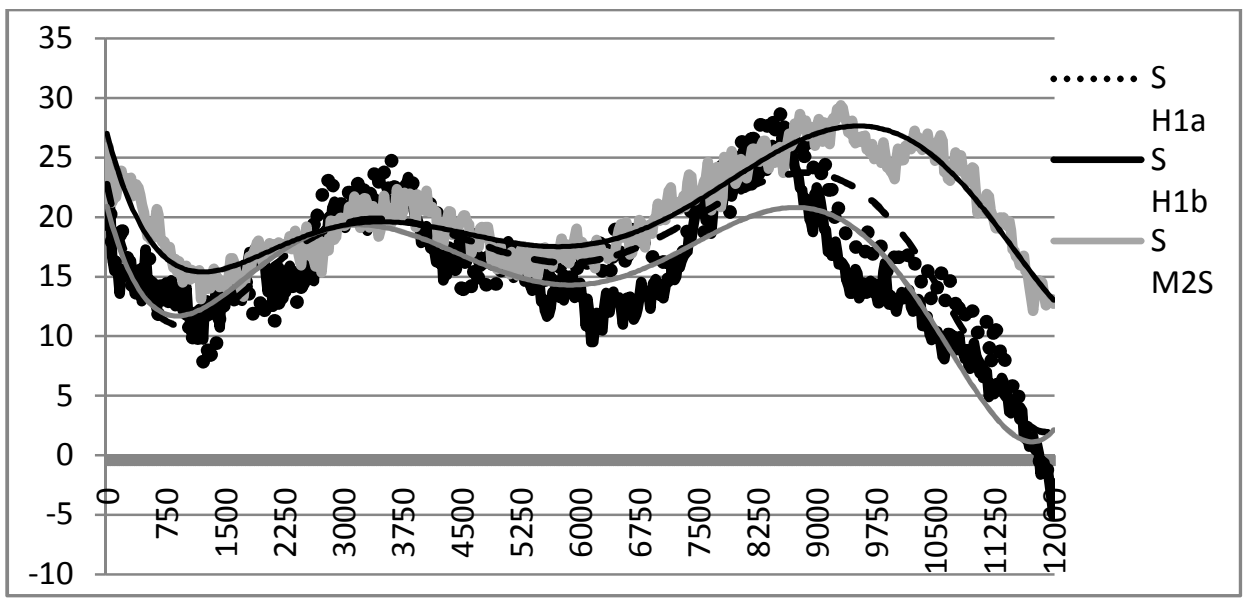

Figura 7. Comparación de los espectros medios para /s/ de los hombres jóvenes y de M2S.

En las hablantes jóvenes de Dumbría, en cambio, no se registra este tipo de perfil espectral, y todas ellas presentan una curva similar, aunque estadísticamente se diferencian dos variantes:

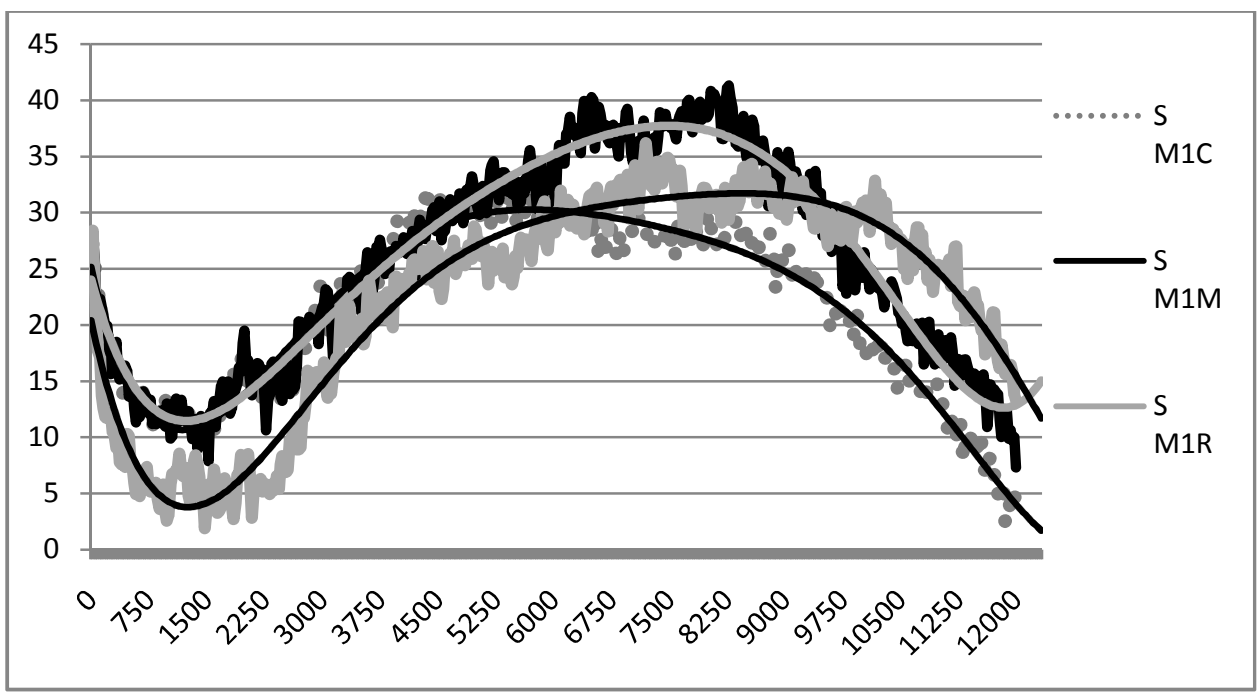

Figura 8. Espectros de /s/ y líneas de tendencia polinómica de las hablantes jóvenes.

Por una parte, la hablante M1C (línea de puntos) presenta un perfil descendente a partir de los $4 \mathrm{KHz}$, y por otro lado las dos restantes (líneas negra y gris), con ascenso hasta los $8 \mathrm{KHz}$ y más allá de los $9 \mathrm{KHz}$. Aunque los perfiles de estas últimas son ligeramente diferentes, las líneas de tendencia polinómica siguen un trazado paralelo, y las series de medias espectrales no presentan diferencias significativas (6942 y 7196 de media, $\mathrm{t}=0.922, \mathrm{p}=0.36$ ). 
La curva de la hablante M1C presenta, en cambio un trazo semejante al de M2C, del grupo de mayor edad. Es cierto que el análisis de centros de gravedad presenta diferencias que estadísticamente son significativas $(p=0.0022)$, por el comportamiento de las bajas frecuencias en $\mathrm{M} 2 \mathrm{C}$, pero ambas se muestran descendentes a partir de 4,5 y $5,25 \mathrm{KHz}$.

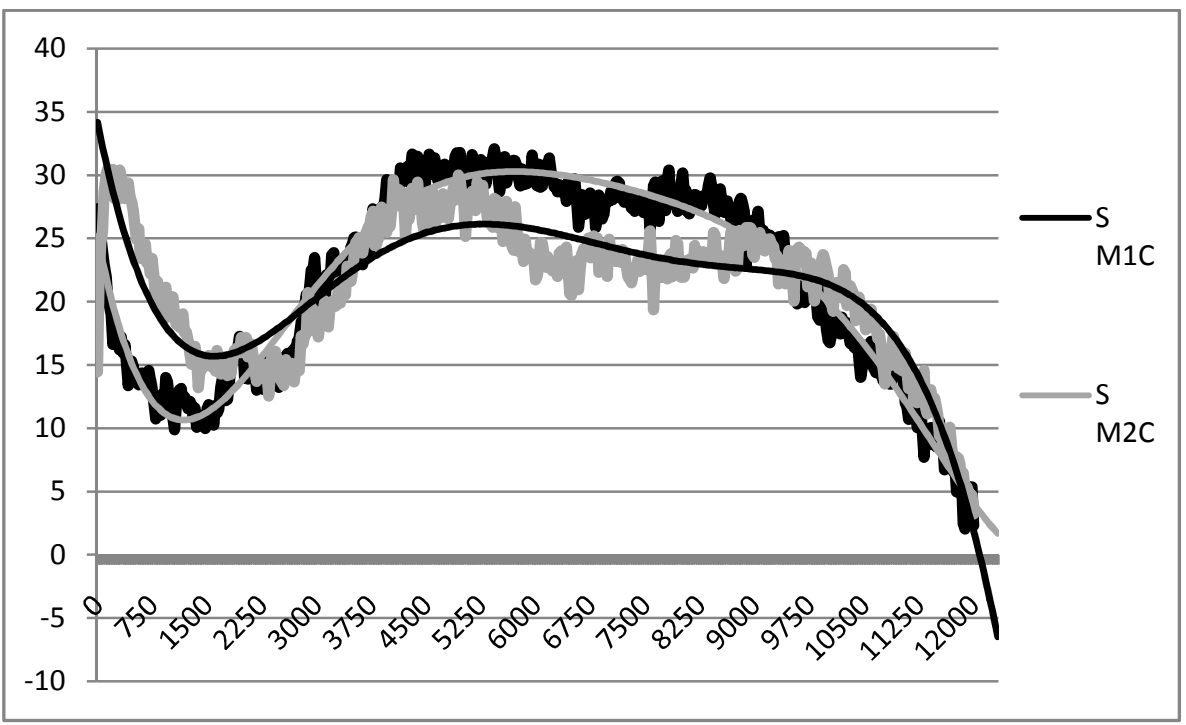

Figura 9. Comparación de los espectros y líneas de tendencia para [s] en las hablantes M1C y M2C.

En cuanto a las sibilantes finales, básicamente se encuentran dos comportamientos diferenciados. Por una parte, el que muestran los hablantes masculinos:

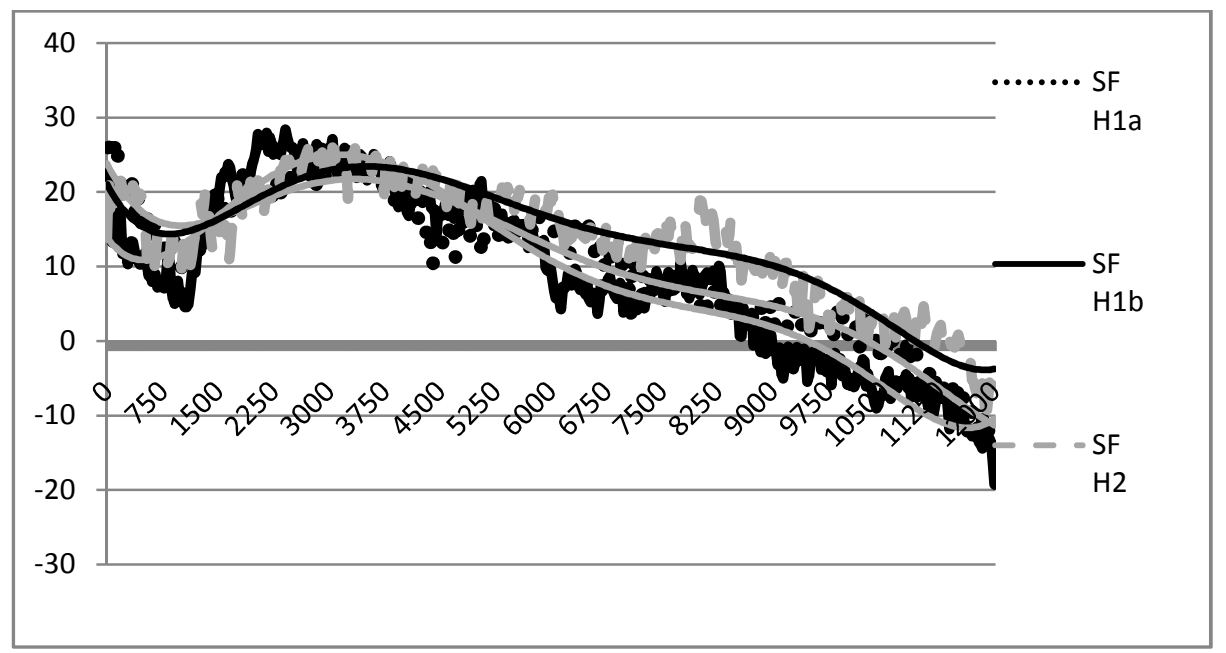

Figura 10. Espectros de [s] en final de palabra en los hablantes masculinos 
Los espectros muestran un perfil descendente a partir de los $2 \mathrm{KHz}$ en los jóvenes, un poco más arriba en el hablante mayor. Se trata de un perfil muy semejante al de la fricativa laminopostalveolar [S]. De hecho, si comparamos las curvas de estas dos realizaciones en el hablante $\mathrm{H} 2$ se puede comprobar esta semejanza:

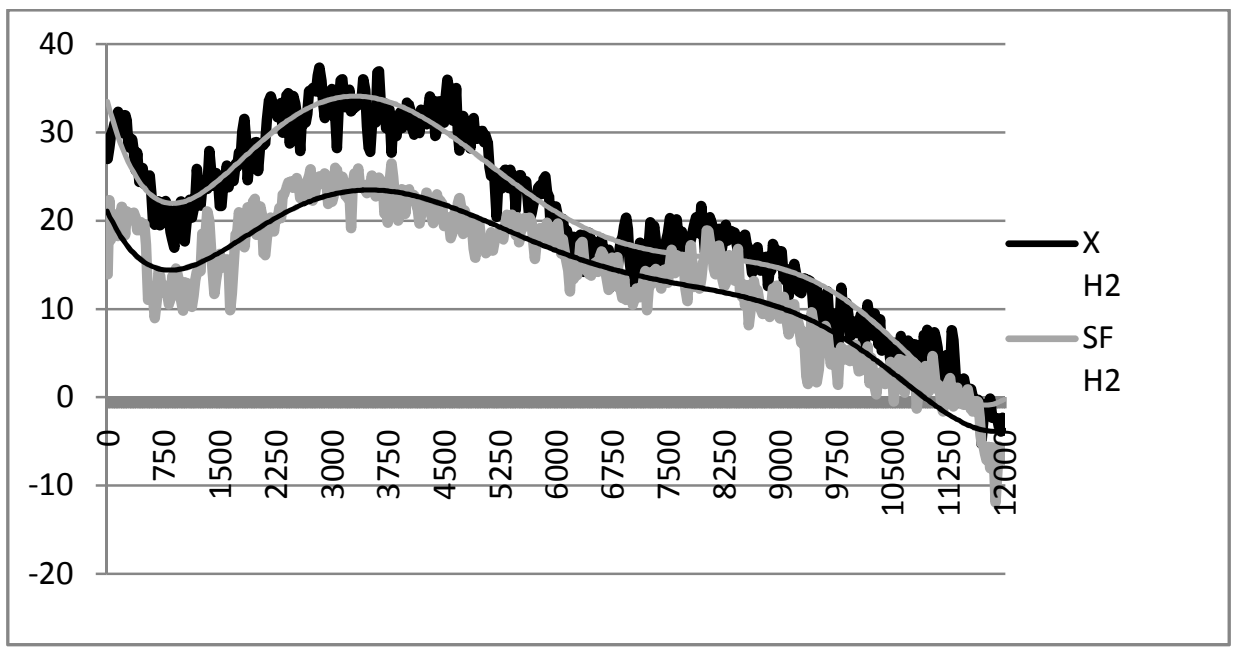

Figura 11. Comparacion e los espectros y líneas de tendencia polinómica para /s/ final y / $/$ en $\mathrm{H} 2$.

La diferencia está en el nivel de energía, mayor en la sibilante en posición inicial de sílaba. En cuanto al reparto de energía, no muestran diferencias significativas (media de centros de gravedad de $3480 \mathrm{~Hz}$ para la sibilante final y 3160 para [S], p=0.36). Tampoco son diferentes en asimetría ( 1 y 0.59 respectivamente, $p=0.17$ ). Estas realizaciones, especialmente las más graves, presentes en los jóvenes, corresponden a una articulación apicopostalveolar [s]. Las informantes femeninas presentan, en cambio, dos comportamientos diferenciados:

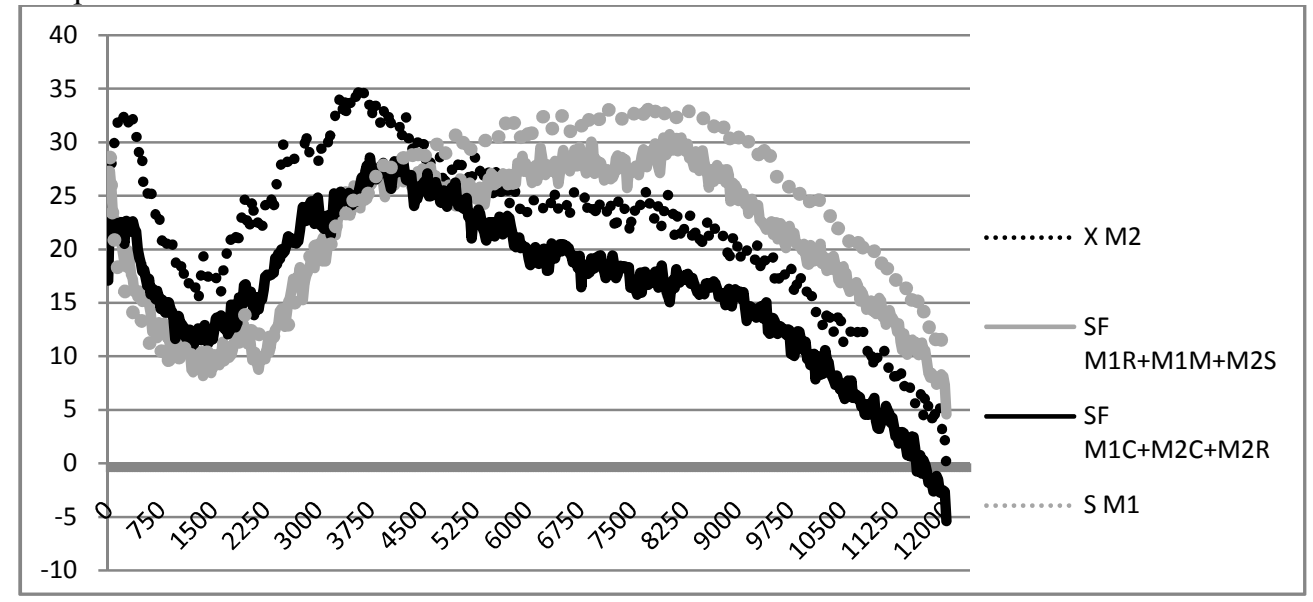

Figura 12. Espectros de /s/ en posición final en las hablantes femeninas. 
En este gráfico se puede apreciar que tres hablantes (línea negra)presentan un perfil descendente, aunque a frecuencia más elevada que los hombres, semejante al de [S] (línea negra de puntos), mientras que otras tres (línea gris) siguen un patrón que se acerca mucho al de [s] (línea gris de puntos). Los análisis estadísticos de centros de gravedad muestran diferencias significativas entre estas dos realizaciones de /s/ final respecto de [s] y de [J], pero los espectros muestran que, mientras que en un caso tenemos sonidos más graves, con pronunciación en la parte posterior de los alvéolos (posiblemente apical), en otro tenemos una fricativa que presenta características acústicas similares a la laminoalveolar [s].

Lo más interesante es que el corte no se produce entre los dos grupos de edad, sino que en el patrón más conservador, desde el punto de vista de este dialecto (véase el comportamiento del grupo masculino), tenemos a dos hablantes del grupo mayor y a una joven, mientras que en el grupo que hace escasa distinción entre posición inicial de sílaba y final de palabra se encuentran las otras dos jóvenes y una hablante del grupo mayor (de 73 años).

\section{Discusión}

Después de analizar los espectros y de tomar en cuenta los centros de gravedad y la asimetría de las curvas resultantes, nos encontramos con comportamientos lingüísticos complejos, y que en pocos casos muestran de manera clara una dirección en cuanto al cambio lingüístico que tratamos de analizar.

Por una parte, se confirma que la fricativa [S] muestra perfiles y valores bastante coherentes en todas las personas que abarca nuestro estudio, mientras que las realizaciones anteriores presentan una notable variación entre hablantes. Básicamente, se pueden diferenciar realizaciones más adelantadas, laminoalveolodentales o laminodentales, y otras laminoalveolares. En posición final, la mayoría de las personas encuestadas muestran realizaciones bastante graves, más semejantes al perfil de [S], que sin duda corresponden a realizaciones apicoalveolares, o en otros casos apicopostalveolares, mientras que tres hablantes femeninas (dos jóvenes y una mayor) presentan realizaciones próximas a $[\mathrm{s}]$.

Teniendo en cuenta el sistema que se ha descrito para este dialecto y los cambios que se han detectado en otros estudios, la primera cuestión que se constata es que en estos grupos de hablantes no se registra ni la «despalatalización» de / $/$ / (aunque algunas realizaciones esporádicas sí causan esa impresión auditiva), ni mucho menos su fusión con la fricativa alveolar. En todos los casos se mantienen dos fonemas y dos series de realizaciones claramente diferenciadas.

En cuanto a la interpretación de los datos relativos a las realizaciones de /s/ en posición inicial de sílaba (inicial de palabra e intervocálica) y en posición final de palabra, las tres mujeres jóvenes presentan unas fricativas semejantes entre sí, relativamente parecidas a las de dos hablantes mayores de su mismo lugar y a las del hablante mayor de Corzón, identificadas como laminoalveolares, mientras que una de las mujeres del grupo mayor (M2S) y los dos jóvenes de Corzón realizan una fricativa más adelantada, que parece corresponder a una articulación laminodental. Esta fricativa es notablemente diferente de las habituales en la lengua estándar (y en el español de Galicia), más atrasadas (y más graves).

Es interesante constatar que M1R es hija de M2R, y que M1C y M1M son hijas de $\mathrm{M} 1 \mathrm{~S}$, pero todas ellas presentan algunas diferencias en las realizaciones fricativas res- 
pecto de sus madres. Tal vez esto sea una muestra de la variabilidad que se produce en las realizaciones de estos sonidos de manera general, pero en todo caso es significativo que todas ellas, con estudios universitarios, aunque con fuertes lazos con su comunidad y con la lengua y cultura de su gente, presenten un modelo de sibilantes menos marcado, aunque con algunos matices (/s/ final en M1C).

Por el contrario, los hablantes jóvenes de Corzón se adscriben a un modelo lingüístico muy diferenciado de la lengua estándar, tanto en las realizaciones de las fricativas en posición inicial de sílaba como en final de palabra, que contrasta con el que muestra el hablante mayor, familiar suyo.

La adscripción de estos dos informantes jóvenes a un modelo lingüístico altamente marcado se revela no solo en las realizaciones de las sibilantes, sino también en otros rasgos, como la alta frecuencia de realizaciones aspiradas de /s/ final de palabra ante consonante en interior de frase de entonación (p.e. tres cans [treh'kãys]), o el mantenimiento de una pronunciación de la gheada con $[\mathrm{h}] \sim[\mathrm{h}]$ (y no con la fricativa velar $[\mathrm{x}]$, como la del español); otro rasgo conservador y muy marcado es el uso de vocal [I] paragógica, como en Esto son... son os coxóns do polo [estu' soyı | 'sõyõhko' fõyhð̛o' polv] (Regueira, en prensa).

Las jóvenes de Dumbría, aunque voluntariamente emplean y mantienen los rasgos fundamentales de la fonología de su variedad nativa (seseo y gheada, notoriamente), muestran una cierta aproximación a las variedades más extendidas y al estándar. Lo que acabamos de ver en la realización de las fricativas sibilantes se corrobora con lo observado en otros aspectos: mientras que M1R mantiene la pronunciación [ $\hbar$ ] que presenta su madre M2R, las otras dos jóvenes, M1C y M1M, pronuncian una fricativa velar [x], que contrasta con la faríngea [ $\hbar]$ de su madre M2S; frente al comportamiento de sus madres, que presentan aspiraciones en la misma posición que los jóvenes de Corzón, aunque en menor medida, las jóvenes no mostraron ni un solo ejemplo de aspiración ni durante la realización de las encuestas ni en 37 minutos de conversación grabada.

El comportamiento de este grupo de jóvenes, así como el de otras personas del grupo de mayor edad, muestra una serie de cambios fonéticos y fonológicos que se van produciendo de manera gradual. Pero también se registra el mantenimiento, menos consciente seguramente, de rasgos fonéticos y fonológicos que configuran una variedad lingüística muy marcada y diferenciada respecto de las lenguas de prestigio. Este hecho muestra que los cambios lingüísticos no se producen de manera lineal de una generación a otra, sino que, del mismo modo que se ha constatado en otras comunidades rurales (p.e. en Escocia, v. McIntosh 1961: 85; Marshall 2004: 4), algunos individuos jóvenes se muestran resistentes al cambio y fuertemente apegados a su variedad propia y no estándar, como un símbolo de pertenencia y de identidad (cfr. Giles et al. 1987: 587; Marshall 2004: 48).

\section{Conclusión}

En este trabajo hemos analizado las realizaciones de las fricativas sibilantes $/ \mathrm{g} / \mathrm{y}$ /s/, en posición inicial de sílaba y final de palabra, en dos grupos de hablantes de un área caracterizada dialectalmente como de «seseo predorsodental». En ella hemos encontrado diferentes fricativas, que van desde una laminodental [s], a laminoalveolar [s], apicoalveolar [s], apicopostalveolar [s], y laminopostalveolar [ $]$ ].

En este estudio hemos diferenciado estas diferentes fricativas por medio del análisis de los espectros de las fricativas y del cálculo del centro de gravedad, y hemos mos- 
trado que son instrumentos eficaces para discriminar realizaciones de sibilantes. De todos modos, distinguir los diferentes tipos de fricativas alveolares a veces resulta difícil, dada la alta variación que se registra entre hablantes, y a veces, incluso entre las realizaciones de una misma persona. No obstante, creemos que los resultados obtenidos son suficientemente claros para establecer los comportamientos generales de cada informante, así como de los grupos establecidos.

Los grupos femeninos no muestran un comportamiento homogéneo en cuanto a las realizaciones de $/ \mathrm{s} /$, lo que hemos interpretado como la constatación de un proceso de cambio gradual, mientras que en el grupo masculino el cambio no se produce, o al menos no se produce en la dirección previsible, y los hablantes jóvenes presentan patrones de sibilantes mucho más caracterizados como diferenciales respecto de la lengua estándar que el hablante mayor.

Estos resultados solo pueden tomarse como indicios, dado que las muestras todavía no son muy numerosas. De todos modos los indicios van en la misma dirección que otros rasgos lingüísticos caracterizadores de las variedades de habla más tradicional, y socialmente marcada, por lo que creemos que deben tomarse como un punto de partida sólido para la continuación y profundización del estudio de los cambios fonéticos en esta área.

Los resultados de este trabajo ponen de manifiesto que los cambios fonéticos en aspectos tan complejos como el de las sibilantes no se producen de manera uniforme y previsible, y que algunos hablantes jóvenes se muestran resistentes al cambio y adoptan rasgos altamente marcados y diferenciales respecto de las variedades lingüísticas más valoradas socialmente. Nuestra hipótesis es que el prestigio encubierto, la identificación de los hablantes con el grupo y la construcción de identidades sociales diferenciadas son factores relevantes a la hora de explicar estas diferencias.

\section{Referencias bibliográficas}

ALGa = Instituto da Lingua Galega (1999). Atlas Lingüístico Galego. Vol. 3: Fonética. A Coruña, Barrié de la Maza.

DIXON, Robert M. W. (1997). The rise and fall of languages. Cambridge, Cambridge University Press.

EVERS, Vincent\& Reetz, HENNING\& LAHIRI, Aditi (1998). «Crosslinguistic acoustic categorization of sibilants independent of phonological status». Journal of Phonetics 26, 345-370.

FERNÁNDEZ REI, Francisco (1991). Dialectoloxía da lingua galega. Vigo, Xerais.

FuCHS, Susanne \& TODA, Martine (2010): «Do differences in male versus female /s/ reflect biological or sociophonetic factors?». In: Susanne Fuchs\& Martine Toda\& Marzena Żygis, eds. Turbulent sounds. An interdisciplinary guide. Berlin, De Gruyter: 281-302.

Giles, Howard\& Hewstone, Miles\& Ryan, Ellen B.\& Johnson, Patricia (1987). «Research on language attitudes». In: Ulrich Ammon et al., eds. Socioinguistics: an international handbook of the science of language and society. Berlin, De Gruyter: 585-597.

GONZÁleZ GONZÁLEZ, Manuel (1991). «Subsistemas de sibilantes do galego actual». In: Dieter Kremer, ed. Actes du XVIII CLPhR (Trier, 1986), vol. 3. Tübingen, Max Niemeyer: 531-548.

- et al. (2003). O galego segundo a mocidade. A Coruña, Real Academia Galega. 
Gordon, Matthew\& BARThMeIER, Paul\& SAnds, Kathy (2002). «A cross-linguistic acoustic study of voiceless fricatives». Journal of the International Phonetic Association32, 2: 141-174.

Hughes, George W. \& Halle, Morris (1956). «Spectral properties of fricative consonants». Journal of the Acoustical Society of America28, 2: 303-310.

IGLESIAS Álvarez, Ana (2002). Falar galego: "no veo por qué”. Aproximación cualitativa á situación sociolingüistica de Galicia. Vigo, Xerais.

JONGMAN, Allard\& WAYLAND, Ratree\& WONG, Serena (2000). «Acoustic carachteristics of English fricatives». Journal of the Acoustical Society of America 108, 3: 1252-1263.

LABRAÑA BARRERO, Sabela (2005). Estudio acústico das consoantes fricativas do galego. Tesis doctoral. Univ. de Barcelona.

MARSHALL, Jonathan (2004). Language change and sociolinguistics. Rethinking social networks. Houndmills, Palgrave.

Martínez Celdrán, Eugenio \& Fernández Planas, Ana M. (2007). Manual de fonética española. Articulaciones y sonidos del español. Barcelona, Ariel.

McINTOSH, Angus (1961). An introduction to a survey of Scottish dialects. Edinburgh, Thomas Nelson.

MedeIRos, António (2006). Dois lados de um rio. Nacionalismo e etnografias na Galiza e Portugal.Lisboa: ICS.

MSG = Real Academia Galega(1992, 2004). Mapa sociolingüístico de Galicia. Vol. 2. A Coruña: RAG, 1992. Mapa socioligüístico de Galicia2004. Vol. 2. A Coruña: RAG, 2004.

Munson, Benjamin\& McDonald, Elizabeth C.\& DeBoe, Nancy L.\& White, Aubrey R. (2006). "The acoustic and perceptual bases of judgments of women and men's sexual orientation from read speech». Journal of Phonetics 34: 202-240.

NetTle, Daniel \& Romaine, Suzanne (2000): Vanishing voices: the extinction of the world's languages. Oxford, Oxford University Press.

NitTrouer, Susan\& StudDerT-Kennedy, Michael\& McGowan, Richard S. (1989). «The emergence of phonetic segments: evidence from the spectral structure of fricative-vowel syllables spoken by children and adults». Journal of Speech and Hearing Research 32: 120-132.

R CORE TEAM (2013). A language and environment for statistical computing. $\mathrm{R}$ Foundation for Statistical Computing, Vienna. http://www.R-project.org/

RegueIRA, Xosé Luís (2009). «Cambios fonéticos e fonolóxicos no galego contemporáneo». Estudos de Lingüística Galega 1: 147-167.

- (2012). Oralidades. Reflexións sobre a lingua falada no século XXI. A Coruña, Real Academia Galega.

- (en prensa). «Factores internos e externos nos cambios fonolóxicos no galego actual». Actes du XXVII CILPR (Nancy, 2013).

RoJO, Guillermo (1981). «Conductas y actitudes lingüísticas en Galicia», Revista Española de Lingüística 11, 2: 269-310.

RouX, Martine (1982). Fala o fistor e faise o día. Algúns aspectos da tradición oral galega na parroquia do Cebreiro. Sada: O Castro.

SCHWARTZ, Martin F. (1968). «Inentification of speakers' sex from voiceless, isolated fricatives».Journal of the Acoustical Society of America43, 5: 1178-1179.

VIDAL FigUEROA, Tiago (1993). «Proposta descritiva das consoantes fricativas alveolodentais dos dialectos galegos». Cadernos de Lingua 7: 5-26. 